\title{
Experimental and/or Genetically Controlled Alterations of the Renal Microsomal Cytochrome P450 Epoxygenase Induce Hypertension in Rats Fed a High Salt Diet
}

\author{
Keiko Makita, * Kihito Takahashi, * Armando Karara, ${ }^{*}$ Harry R. Jacobson, ${ }^{\star}$ John R. Falck, ${ }^{\S}$ and Jorge H. Capdevila*‡ \\ Departments of $*$ Medicine and ${ }^{\ddagger}$ Biochemistry, Vanderbilt University Medical School, Nashville, Tennessee $37232 ;$ and ${ }^{\S}$ Department of \\ Molecular Genetics, Southwestern Medical Center, Dallas, Texas 75235
}

\begin{abstract}
Excess dietary salt induces a cytochrome $\mathbf{P 4 5 0}$ arachidonic acid epoxygenase isoform in rat kidneys (Capdevila, J. H., S. Wei, J. Yang, A. Karara, H. R. Jacobson, J. R. Falck, F. P. Guengerich, and R. N. Dubois. 1992. J. Biol. Chem. 267:21720-21726). Treatment of rats on a high salt diet with the epoxygenase inhibitor, clotrimazole, produces significant increases in mean arterial blood pressure $(122 \pm 2$ and $145 \pm 4 \mathrm{mmHg}$ for salt and salt- and clotrimazole-treated rats, respectively). The salt- and clotrimazole-dependent hypertension is accompanied by reductions in the urinary excretion of epoxygenase metabolites and by a selective inhibition of the renal microsomal epoxygenase reaction. The prohypertensive effects of clotrimazole are readily reversed when either the salt or clotrimazole treatment is discontinued. The indication that a salt-inducible renal epoxygenase protects against hypertension, are supported by studies with the Dahl rat model of genetic salt-sensitive hypertension. Dahl resistant animals responded to excess dietary salt by inducing the activity of their kidney microsomal epoxygenase $(s)(0.102 \pm 0.01$ and $0.240 \pm 0.04$ nmol of products formed/min per $\mathrm{mg}$ of microsomal protein for control and salt-treated rats, respectively). Despite severe hypertension during excess dietary salt intake $(200 \pm 20 \mathrm{mmHg})$, Dahl salt-sensitive rats demonstrated no increase in renal epoxygenase activity.

These studies indicate that acquired or inherited abnormalities in renal epoxygenase activities and/or regulation can be related to salt-sensitive hypertension in rodents. Studies on the human renal epoxygenase and its relationship to salt hypertension may prove useful. (J. Clin. Invest. 1994. 94:2414-2420.) Key words: arachidonic acid - epoxyeicosatrienoic acids • sodium chloride $\bullet$ blood pressure $\bullet$ Dahl rats
\end{abstract}

\section{Introduction}

Arachidonic acid serves as the precursor for a variety of potent mediators of cell and organ function, including prostanoids and

Portions of this work were presented at the 26th Annual Meeting of the American Society of Nephrology, 14-17 November 1993, Baltimore, MD.

Address correspondence to Jorge H. Capdevila, Vanderbilt University Medical School, Medical Center North S-3223, Nashville, TN 37232.

Received for publication 31 May 1994 and in revised form 22 August 1994.

J. Clin. Invest.

(c) The American Society for Clinical Investigation, Inc. 0021-9738/94/12/2414/07 \$2.00

Volume 94, December 1994, 2414-2420 leukotrienes (1,2, and references therein). More recently, a considerable body of evidence has demonstrated that microsomal cytochrome $\mathrm{P} 450$ also participates in the bioactivation of arachidonic acid (3-5, and references therein). The rat kidney microsomal cytochrome $\mathrm{P} 450$ arachidonic acid epoxygenase catalyzes the NADPH-dependent, enantiospecific, formation of 5,6-, 8,9-, 11,12-, and 14,15-epoxyeicosatrienoic acids (EET) ${ }^{1}$ (5). To date, epoxygenase activity has been demonstrated in numerous rodent and human tissues, including liver, kidney, brain, pituitary, adrenal, ovaries, lung, and endothelium (3-6). Interest in defining the functional significance of the cytochrome P450 epoxygenase has been stimulated by, among other things, the demonstration of a role for this enzyme in the in vivo metabolism of arachidonic acid and by the potent in vitro and in vivo biological activities of its metabolites (3-5). Among the biological activities attributed to the EET or to their hydration products, the corresponding vic-dihydroxyeicosatrienoic acids (DHET), those of significance to the renal regulation of salt and water balance include $(a)$ inhibition of $\mathrm{Na}^{+}$reabsorption and $\mathrm{K}^{+}$secretion in the rabbit cortical collecting tubule (7), (b) inhibition of $\mathrm{Na}^{+}$transport by the proximal tubule (8), (c) inhibition of vasopressin-stimulated water reabsorption in the collecting tubule $(9,10),(d)$ enantioselective intrarenal vasoconstriction (11) and, $(e)$ modulation of renal $\mathrm{Na}^{+}, \mathrm{K}^{+} \mathrm{AT}$ Pases (12).

In normal Sprague-Dawley rats excess dietary salt results in marked increases in the urinary excretion of epoxygenase metabolites (13). Metabolic and immunological evidence demonstrated that a high salt diet induces a cytochrome P450 isoform which is either absent or present at very low concentrations in the kidneys of untreated animals (13). Increased salt intake results in increased renal salt excretion. This efficient adaptive process prevents progressive salt retention, volume expansion and, one of its detrimental sequelae, hypertension. The association between an increased salt intake and the marked increases in renal epoxygenase activity and urinary excretion of EET and DHET, in conjunction with the known functional effects of the EET as inhibitors of proximal and distal nephron $\mathrm{Na}^{+}$absorption $(4,7,8)$, suggested that the salt-inducible cytochrome $\mathbf{P} 450$ arachidonic acid epoxygenase may be one of the functionally significant components of the kidney's adaptive response to an increased salt intake.

Earlier studies with the spontaneously hypertensive rat model (SHR/WKY) indicated that the renal cytochrome P450 arachidonic acid monooxygenase system may be of importance

1. Abbreviations used in this paper: DHET, vic-dihydroxyeicosatrienoic acid; DR, Dahl salt-resistant genotype; DS, Dahl salt-sensitive genotype; EET, cis-epoxyeicosatrienoic acid; MABP, mean arterial blood pressure; NO, nitric oxide; SHR, spontaneously hypertensive rat. 
in the pathophysiology of hypertension (4). Thus, in this rat model, the developmental phase of hypertension was temporally linked to attendant increases in the activities of the kidney microsomal $\omega / \omega$-1 oxygenase (4). More recently, the gene coding for cytochrome P450 4A2 was shown to be preferentially expressed in SHR animals (14). The demonstration that $(a)$ the cytochrome P450 arachidonic acid epoxygenase is a member of the endogenous arachidonic acid metabolic cascade in human and rodent kidneys, $(b)$ in humans, pregnancy induced hypertension results in substantial increases in the urinary excretion of DHTE (15), and (c) the renal epoxygenase is regulated by excess dietary salt (13) further supports the proposal that this enzyme system may be a participant in the mechanisms by which the kidneys control body salt and fluid composition.

\section{Methods}

Animals. Male Sprague-Dawley rats (300-350 g body wt) (Harlan Sprague Dawley, Inc., Indianapolis, IN) or male Dahl salt-resistant (Hsd:DR) and Dahl salt-sensitive (Hsd:DS) rats (280-320 $\mathrm{g}$ body wt) (outbread, Brookhaven National Laboratories, Long Island, NY) (Harlan Sprague Dawley, Inc.) were used for these experiments. Animals, maintained at $22^{\circ} \mathrm{C}$ with alternating 12 -h cycles of light and dark, were allowed free access to water and fed either standard rat chow containing $\sim 0.4 \%$ sodium (wt/wt) (equivalent to $1 \% \mathrm{NaCl} w t / w t$ ) or a modified lab chow containing $8 \% \mathrm{NaCl}$ (wt/wt) (special mix 5001-2; Ralston Purina Mills, St. Louis, MO). Studies of the functional role of the epoxygenase were done using groups of age-matched rats ( $\geq 10$ animals per group) treated according to the following protocols ( $a$ ) group A, fed a standard laboratory diet containing $0.4 \%$ sodium (wt/wt) for 37 $\mathrm{d},(b)$ group B, fed the same diet as group A for $37 \mathrm{~d}$ but, after $35 \mathrm{~d}$, the animals were injected twice with clotrimazole $(80 \mathrm{mg} / \mathrm{kg}$ body wt, i.p., once every $24 \mathrm{~h}$ ), (c) group C, fed a high salt diet containing $8 \%$ $\mathrm{NaCl}(\mathrm{wt} / \mathrm{wt}$ ) for $37 \mathrm{~d}$ and, $(d)$ group $\mathrm{D}$, fed the same diet as group $\mathrm{C}$ for a total of $37 \mathrm{~d}$ but, after $35 \mathrm{~d}$, the animals were injected twice with clotrimazole ( $80 \mathrm{mg} / \mathrm{kg}$ body wt, i.p., once every $24 \mathrm{~h}$ ). At the end of the 37-d period, urine samples were collected over an 8-12-h period and, after light anesthesia, the animals mean arterial blood pressure (MABP) was measured. Clotrimazole $(80 \mathrm{mg} / \mathrm{kg}$ body wt, i.p.) (Sigma Chemical Co., St. Louis, MO) was administered, suspended in corn oil, once every $24 \mathrm{~h}$. For MABP measurements, animals were anesthetized (Inactin, $100 \mathrm{mg} / \mathrm{kg}$, i.p.), a catheter $(0.025 \mathrm{~mm}$ in diameter) was then surgically inserted into the femoral artery and systemic blood pressures were monitored $(1-3 \mathrm{~h})$ by means of a pressure transducer and a recorder. Immediately thereafter, the animals were killed, under anesthesia, by heart puncture, their kidneys immediately removed, freed of connective tissue and utilized for the isolation of microsomal fractions as described below (16). To confirm the Dahl animals saltsensitive or resistant phenotypes, MABP were determined by the tail cuff method using a blood pressure analyzer instrument (model 178, IITC, Life Science USA, Woodland Hills, CA), at $30^{\circ} \mathrm{C}$ and following the manufacturer's instructions. Animals were exposed to the $30^{\circ} \mathrm{C}$ chamber for at least 30 min before measurements. MABP was obtained after four consecutive readings whose values were within $\pm 5 \%$ of their mean.

Microsomal incubations. Microsomal fractions were isolated exactly as previously described (16). Briefly, whole kidneys, obtained from control or salt-loaded animals, were collected on ice, minced, and immediately homogenized in $0.05 \mathrm{M}$ Tris- $\mathrm{Cl}$ ( $\mathrm{pH} 7.5$ ) containing $0.25 \mathrm{M}$ sucrose using a glass/teflon grinder. Microsomal fractions, isolated by differential centrifugation, were suspended $(1 \mathrm{mg}$ of protein $/ \mathrm{ml}$, final concentration) in $0.05 \mathrm{M}$ Tris- $\mathrm{Cl}$ buffer ( $\mathrm{pH} \mathrm{7.5)}$ containing $0.15 \mathrm{M}$ $\mathrm{KCl}, 10 \mathrm{mM} \mathrm{MgCl}, 8 \mathrm{mM}$ sodium isocitrate and $0.5 \mathrm{IU}$ of isocitrate dehydrogenase and, incubated with $\left[1-{ }^{14} \mathrm{C}\right]$ arachidonic acid $(1.0-2.0$ $\mu \mathrm{Ci} / \mu \mathrm{mol}, 100 \mu \mathrm{M}$, final concentration) in the presence of $1 \mathrm{mM}$ $\mathrm{NADPH}$ and at $30^{\circ} \mathrm{C}$. Aliquots of the reaction mixtures were withdrawn

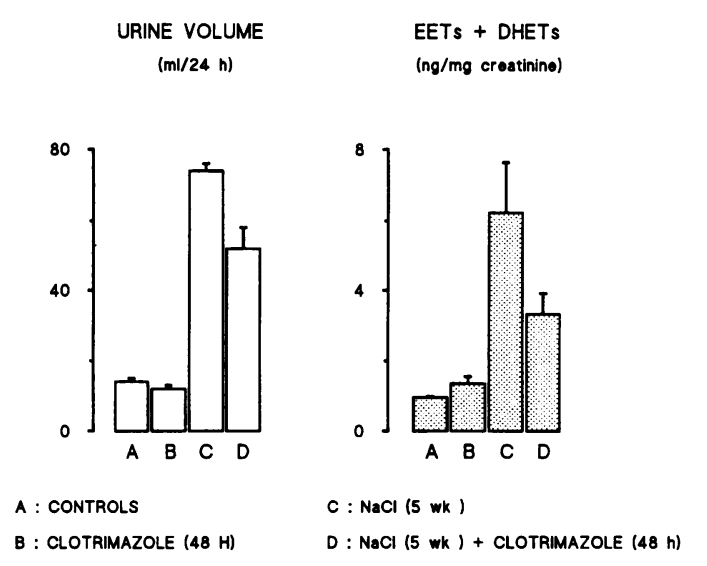

Figure 1. Changes in urine volume and urinary excretion of epoxygenase metabolites after the administration to rats of either clotrimazole, a high salt diet, or a combination of both. Groups of male Sprague-Dawley rats (300-350 $\mathrm{g}$ body wt) were fed standard rat chow containing $0.4 \%$ sodium (wt/wt) $(A)$ or a modified lab chow containing $8 \% \mathrm{NaCl}$ (wt/ wt) (special mix 5001-2; Purina) (C) for a total of $37 \mathrm{~d}$. After $35 \mathrm{~d}$, a group of control $(B)$ and salt-loaded rats $(D)$ was injected twice with a suspension of clotrimazole in corn oil $(80 \mathrm{mg} / \mathrm{kg}$ body wt, i.p., once every $24 \mathrm{~h}$ ). Urine samples were collected over an 8-12 h period and, after volume measurements, the urinary concentration of EET and DHET was determined as described in Methods. Values are averages $\pm S E$ of the mean, calculated from at least seven different experiments.

at different time points, the reaction products extracted into ethyl ether (containing $0.05 \% \mathrm{HOAc}$ ) and, after solvent evaporation, resolved by reversed phase HPLC (16). Products were quantified by on-line liquid scintillation utilizing a Radiomatic Flo-One $\beta$-Detector (Radiomatic Instruments, Tampa, FL). Microsomal fractions were stored at $4^{\circ} \mathrm{C}$ and used within $24 \mathrm{~h}$ of isolation. Initial reaction rates, obtained from the linear portion of reaction rate plots, are given as nmol of product formed/ $\mathrm{min}$ per $\mathrm{mg}$ of microsomal protein $\pm \mathrm{SE}$.

Analytical procedures. For negative ion chemical ionization/gas chromatography/mass spectral analysis and quantification, urine samples were collected, overnight (8-12-h periods), over $5-10 \mathrm{mg}$ of triphenylphosphine $(13,17)$. Each individual sample was mixed, filtered, and $10-20-\mathrm{ml}$ aliquots were then withdrawn for analysis. After acidification and extraction with 2 vol of $\mathrm{CHCl}_{3} / \mathrm{CH}_{3} \mathrm{OH}(2: 1)$, the urinary concentration of EET and DHET was determined by a combination of the isotope dilution/gas chromatography/mass spectroscopic techniques previously described $(13,17)$. Urine creatinine was measured using a Sigma Diagnostics Creatinine Kit (Sigma Chemical Co.) and the manufacturer's instructions. Sodium concentrations were determined by flame photometry.

\section{Results}

To ascertain the functional significance of the induction of the kidney cytochrome P450 arachidonic acid epoxygenase in response to excess dietary salt, groups of age-matched rats were treated with salt and clotrimazole, an arachidonic acid epoxygenase inhibitor (18), as indicated in Methods. From each group of animals 8-12 h urine samples were collected and, after light anesthesia, their MABP measured. As shown in Fig. 1, compared with control animals (group A), clotrimazole treatment (group B) resulted only in minor changes in urine volume or in the urinary excretion of epoxygenase metabolites. In contrast, increased dietary salt (group C) induced a 5-6-fold increase in urine volume and, as previously reported (13), a 


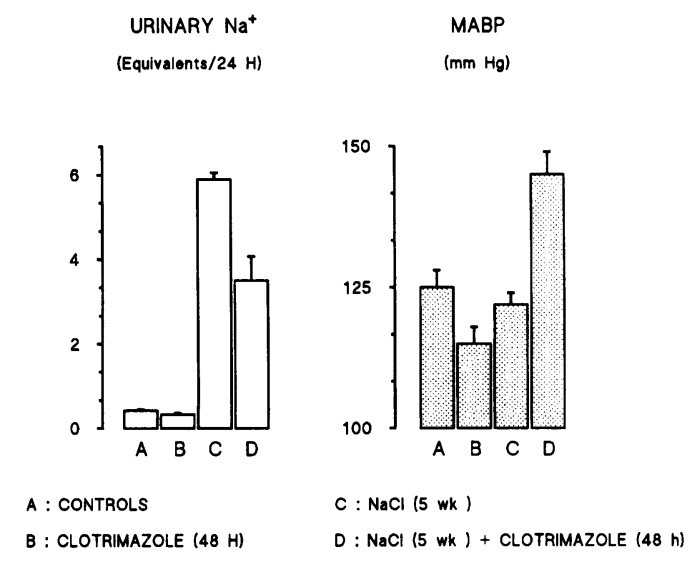

Figure 2. Changes in the urinary excretion of sodium and in mean arterial blood pressure of rats administered either clotrimazole, excess salt, or a combination of both. For these experiments, measurements were performed using the same groups of animals used in Fig. 1. Sodium concentrations were determined by flame photometry. MABP was measured as described in Methods. Animals were then killed, under anesthesia, by heart puncture, their kidneys removed free of connective tissue and used for the isolation of microsomal fractions as described in Methods.

concomitant 6-7-fold increase in the urinary excretion of epoxygenase metabolites (Fig. 1). Within $48 \mathrm{~h}$ of clotrimazole treatment, the salt-loaded animals (group D) showed significant decreases in urine volume $(74 \pm 2$ and $52 \pm 6 \mathrm{ml} / \mathrm{d}$ for salt and salt plus clotrimazole treated, respectively; $n=5$ ) (Fig. 1) and in the urinary excretion of EET and DHET (6.2 \pm 1.4 and $3.3 \pm 0.6 \mathrm{ng} / \mathrm{mg}$ of creatinine for salt and salt plus clotrimazole treated, respectively; $n=5$ ) (Fig. 1 ).

The effects of inhibition of the cytochrome P450 arachidonate epoxygenase on $\mathrm{Na}^{+}$excretion and arterial blood pressure are shown in Fig. 2. After two daily injections of clotrimazole, animals fed $0.4 \%$ sodium diets showed no change in their urinary $\mathrm{Na}^{+}$excretion $(0.42 \pm 0.03$ and $0.33 \pm 0.03$ equivalents/ $24 \mathrm{~h}$ for control (A) and clotrimazole treated animals (B), respectively, $n=6$ ). In these animals, mean arterial blood pressure (MABP) fell slightly $(125 \pm 3$ and $115 \pm 3 \mathrm{mmHg}$ for control (A) and clotrimazole treated (B), respectively; $n=5$ ) (Fig. 2). On the other hand, the animals fed a high salt diet (C) responded by increasing their urinary $\mathrm{Na}^{+}$excretion nearly 14-fold ( $5.89 \pm 0.16$ equivalents $/ 24 \mathrm{~h} n=9$ ). Dietary salt loading did not result in a significant elevation of MABP [122 \pm 2 $\mathrm{mmHg}$ in salt loaded (C) vs $125 \pm 3 \mathrm{mmHg}$ in controls (A), $n$ $=5$ ] (Fig. 2). However, in animals fed a high salt diet and treated with the cytochrome P450 inhibitor (group D), urinary $\mathrm{Na}^{+}$excretion was reduced almost by half $(3.50 \pm 0.57$ equivalents/24 h, $n=6$ ) while the MABP of these animals increased to $145 \pm 4 \mathrm{mmHg}(n=9)$ (Fig. 2$)$. Importantly, animals became hypertensive only after the combined effects of salt loading and clotrimazole treatment (Fig. 2). When either the clotrimazole treatment was stopped or the high salt diet was replaced by regular, $0.4 \%$ sodium chow, the blood pressure of the hypertensive animals (group D) returned to normal (within $2 \mathrm{~d}$ after cessation of clotrimazole treatment or, alternatively, within 5$6 \mathrm{~d}$ after removal of the high salt diet).

To demonstrate that the reduced urinary excretion of EET and DHET after clotrimazole treatment was due to inhibition

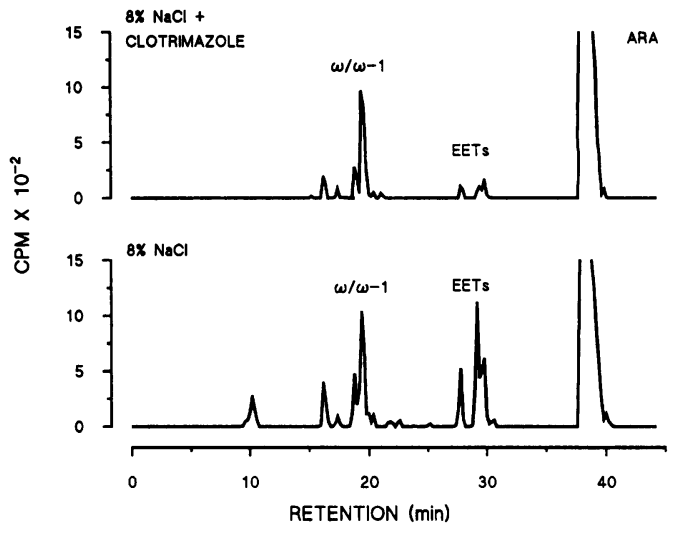

Figure 3. Effect of clotrimazole administration on the kidney microsomal arachidonic acid epoxygenase activity of salt-loaded rats. Microsomal fractions ( $1 \mathrm{mg}$ of protein $/ \mathrm{ml}$, final concentration) isolated from the kidneys of salt-loaded, and salt-loaded and clotrimazole-treated animals (Figs. 1 and 2, groups $\mathrm{C}$ and $\mathrm{D}$ ), were incubated, at $30^{\circ} \mathrm{C}$, with [1- $\mathrm{C}^{14}$ ] arachidonic acid $(2.0-2.5 \mu \mathrm{Ci} / \mu \mathrm{mol}, 100 \mu \mathrm{M}$, final concentration) in the presence of $1 \mathrm{mM}$ NADPH. The reaction products were resolved and quantified by on-line liquid scintillation as described in Methods. Shown are the reversed phase HPLC profiles of the metabolites generated, after a 20 -min incubation period, by incubates containing microsomal fractions isolated from either salt-loaded (group C) (bottom), or salt-loaded and clotrimazole-treated animals (group D) (top) (1 $\mathrm{mg}$ of microsomal protein, each). Shown in the chromatograms are the approximate retention times for a mixture of 19- and 20-hydroxyeicosatetraenoic acids $(\omega / \omega-1)$ and of 14,15-, 11,12-, 8,9-, and 5,6-epoxyeicosatrienoic acids (EET).

of the endogenous kidney epoxygenase, kidney microsomal fractions, isolated from the kidneys of hypertensive, high saltand clotrimazole-treated animals (group D), as well as from the high salt, normotensive (group C), were incubated with [1${ }^{14} \mathrm{C}$ ] arachidonic acid $(100 \mu \mathrm{M})$ and NADPH (1 mM) (16). After $10-20 \mathrm{~min}$ at $30^{\circ} \mathrm{C}$, the reaction products were extracted and resolved by reversed phase HPLC (16). As is evident from the chromatograms in Fig. 3, animal treatment with clotrimazole resulted in a marked and selective inhibition of the epoxygenase reaction ( $\leq 15 \%$ of control rates, $n=4$ ) with little or no effect on the microsomal $\omega / \omega-1$ arachidonate oxygenase ( $\geq 90 \%$ of control rates, $n=4$ ). This confirmed earlier studies with rat liver microsomes where clotrimazole was shown to be epoxygenase specific (18).

Using an experimental protocol identical to the one described above, we showed that administration of the cyclooxygenase inhibitor, indomethacin ( $80 \mathrm{mg} / \mathrm{kg}$ body wt, i.p.), had no significant effects on the systemic blood pressure of saltloaded animals. Also, and as previously shown (19), NG-monomethyl-L-arginine, an NO-synthase inhibitor (20), administered as a $0.2 \%$ (wt/vol) solution in the drinking water for $10 \mathrm{~d}$ caused a marked increase in the animals MABP (115 \pm 15 and $180 \pm 20 \mathrm{mmHg}$ for control and NG-monomethyl-L-argininetreated animals, respectively). In these animals, the arginine analogue did not alter the activity of the kidney microsomal arachidonic acid epoxygenase or $\omega / \omega-1$ oxygenase, nor did it induce significant changes in the urine or plasma concentrations of epoxygenase metabolites. Importantly, the prohypertensive effect of NG-monomethyl-L-arginine was independent of salt loading and/or clotrimazole treatment.

These results strongly support the hypothesis that the kidney 
(A) DAHL RATS, $0.4 \times \mathrm{Na}^{+}$FOOD

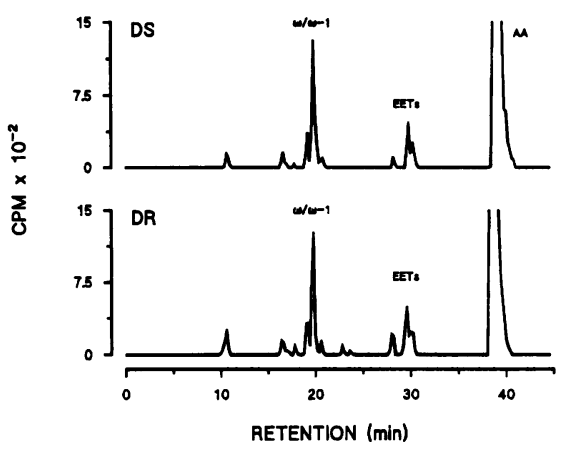

(B) DAHR RATS, 8X NaCI FOOD

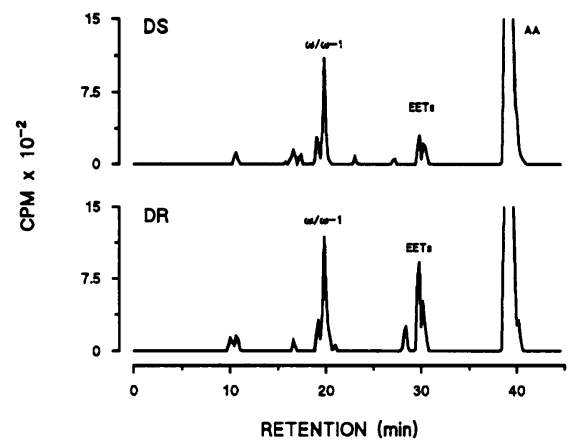

Figure 4. Effect of salt loading on the catalytic properties of the microsomal arachidonic acid monooxygenase system present in the kidneys of salt-sensitive (DS) and salt-resistant Dahl (DR) rats. Male DR and DS rats were fed either standard $(0.4 \%$ sodium wt/ wt) or a modified lab chow ( $8 \%$ $\mathrm{NaCl}$, wt/wt) for 16-20 d. Animals were then killed and whole kidney microsomal fractions were isolated as described in Methods. The catalytic properties of the microsomal fractions isolated from animals maintained either in the low salt (equivalent to $1 \%$ $\mathrm{NaCl} \mathrm{wt} / \mathrm{wt})(A)$ or high salt diets $(8 \% \mathrm{NaCl} w t / w t)(B)$ were analyzed using the incubation conditions and the analytical methodology described in Fig. 3. The radiochromatograms shown were generated by incubating $0.75 \mathrm{mg}$ of microsomal protein at $30^{\circ} \mathrm{C}$ for $20 \mathrm{~min}$. Shown in the chromatograms are the approximate retention times for a mixture of 19- and 20-hydroxyeicosatetraenoic acids $(\omega / \omega-1)$ and of 14,15-, 11,12-, 8,9-, and 5,6-epoxyeicosatrienoic acids (EET).

microsomal arachidonic acid epoxygenase plays a functionally significant role in the control of $\mathrm{Na}^{+}$homeostasis and systemic blood pressure. To further explore the potential links between dietary salt, blood pressure, and the renal cytochrome P450 epoxygenase we used the Dahl rat model of genetic salt-dependent hypertension. In these animals, genetically controlled alterations in renal $\mathrm{Na}^{+}$metabolism lead to the development of systemic hypertension after salt loading (21 and references therein, and 22). For these studies, groups of DS and DR rats (280-300 $\mathrm{g}$ body wt) were fed either a standard laboratory rat chow $(0.4 \%$ sodium) or a modified high salt diet $(8 \% \mathrm{NaCl})$ for 2-3 wk. At the end of this time period, systemic blood pressures were measured, the animals killed, their kidneys removed, microsomal fractions isolated and incubated with [1${ }^{14} \mathrm{C}$ ] arachidonic acid in the presence of NADPH. After extraction, the reaction products were resolved by reversed phase HPLC (16). No significant differences in the rates of total arachidonic acid metabolism were observed by incubating microsomal fractions obtained from either the DR (MABP $=120 \pm 10 \mathrm{mmHg})$ or DS $($ MABP $=130 \pm 15 \mathrm{mmHg})$ animals fed the low salt diet (Fig. $4 \mathrm{~A}$ ) (Table I). As with SpragueDawley animals (13), the DR rats responded to the high salt

Table I. Effect of Salt Loading on the Catalytic Properties of the Microsomal Arachidonic Acid Monooxygenase Present in the Kidneys of Salt-sensitive (DS) and Salt-resistant (DR) Dahl Rats

\begin{tabular}{lccc}
\hline Source of enzymes & Total metabolism & Epoxygenase & $\omega / \omega-1$ Oxygenase \\
\hline DR control & $0.289 \pm 0.03$ & $0.102 \pm 0.01$ & $0.188 \pm 0.02$ \\
DR NaCl & $0.430 \pm 0.05$ & $0.240 \pm 0.04$ & $0.180 \pm 0.03$ \\
$D S$ control & $0.276 \pm 0.04$ & $0.082 \pm 0.01$ & $0.189 \pm 0.02$ \\
$D S \mathrm{NaCl}$ & $0.268 \pm 0.05$ & $0.098 \pm 0.02$ & $0.168 \pm 0.02$
\end{tabular}

Whole kidney microsomal fractions were isolated from the kidneys of male DR and DS Dahl rats fed either standard ( $0.4 \%$ sodium, wt/wt) or a modified lab chow (8\% $\mathrm{NaCl}$, wt/wt) for 16-20 d. Microsomal fractions were incubated at $30^{\circ} \mathrm{C}$ in the presence of $100 \mu \mathrm{M}\left[1-{ }^{14} \mathrm{C}\right]$ arachidonic acid and $1 \mathrm{mM}$ NADPH as described in Fig. 3 and in Methods. Values shown are initial velocities expressed as nmol of product formed/ $\mathrm{mg}$ of microsomal protein/min. Shown are the averages $\pm \mathrm{SE}$ of the mean, calculated from at least six different experiments. diet with an overall increase in their renal arachidonic acid oxygenase activity (149\% of the rates obtained with control, non-salt-treated rats) (Table I). These salt induced changes could be almost completely accounted for by a salt-dependent $235 \%$ increase in the kidney epoxygenase activity of DR rats (Table I). Importantly, during the course of salt loading, the DR animals remained essentially normotensive (MABP $=130 \pm 10$ $\mathrm{mmHg}$ ). In contrast, under the same experimental conditions, the DS animals were unable to induce their renal microsomal epoxygenase activity in response to salt loading (Table I) (Fig. $4 B$ ). The MABP of the DS animals increased rapidly and became, more or less stable, 14-17 d after the initiation of salt loading $(200 \pm 20 \mathrm{mmHg})$. No significant phenotypic differences and/or dietary alterations in the microsomal $\omega / \omega-1$ arachidonate oxygenase of the DS or DR animals were observed (23) (Table I) (Fig. 4, $A$ and $B$ ).

To substantiate the in vivo significance of these observations, groups of DR and DS animals were allowed to drink either water or a $2 \%$ solution of $\mathrm{NaCl}$ for 7-10 d and, after extraction and purification, the concentration of epoxygenase metabolites in their urine was measured by GC/MS techniques (17). To improve reproducibility a $2 \% \mathrm{NaCl}$ solution, instead of the solid diet containing $8 \% \mathrm{NaCl}$, was used as the source of high dietary salt (13). As with Sprague-Dawley animals, excess dietary salt resulted in an increased urinary excretion of epoxygenase metabolites (expressed as ng of DHET $/ \mathrm{mg}$ of creatinine) by both DR and DS rats (Fig. 5). However, while for DS animals the excretion of individual epoxygenase metabolites was approximately doubled, with the DR animals salt induced 9-, 8-, 7- and 4-fold increases in the urinary excretion of 8,9-, 11,12-, 14,15-, and 5,6-EET + DHET, respectively (Fig. 5). Importantly, when compared to nontreated controls, excess dietary salt resulted in significant decreases in the urine concentration of epoxygenase metabolites (expressed as $\mathrm{ng}$ of product/ $\mathrm{ml}$ of urine) only in the DS animals (Fig. 5). On the other hand, the urine of salt-treated DR rats showed significant increases in the levels of epoxygenase products (Fig. 5). It is of interest that the kidney microsomal epoxygenase activities and the isomeric composition of the urinary epoxygenase metabolites are similar for control DR and DS animals (Tables I and II), thus further illustrating that the observed phenotypic differences between the Dahl DR and DS genotypes are manifested only after excess dietary salt. 


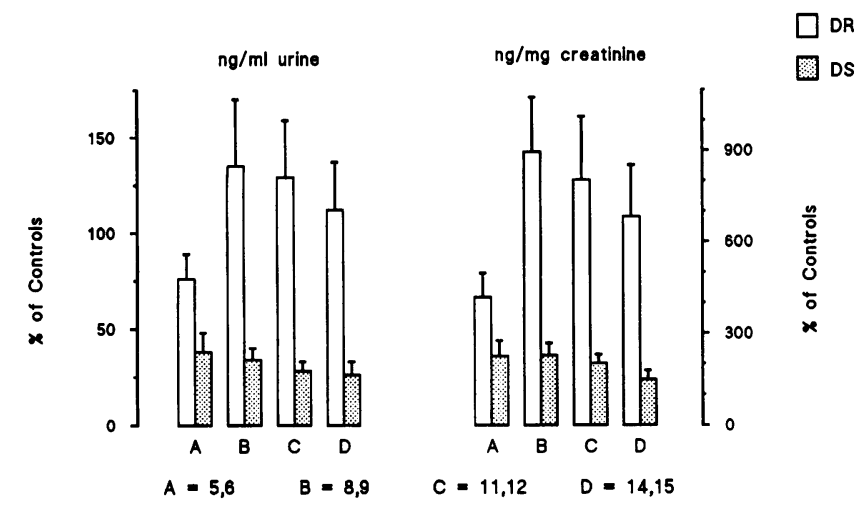

Figure 5. Changes in the urinary concentrations of epoxygenase metabolites after the administration of high salt to salt-sensitive (DS) and saltresistant (DR) Dahl rats. Groups of male DR and DS rats (280-300 g body $\mathrm{wt}$ ) were allowed to drink freely either water or a $2 \%$ solution of $\mathrm{NaCl}$ for 7-8 d. Urine samples were collected over triphenylphosphine for 8-12 $\mathrm{h}$ and, after extraction and purification, their EET and DHET contents determined by gas chromatography/mass spectrometry techniques as described (17). Salt-induced changes in the urine concentration of epoxygenase metabolites in $\mathrm{ng} / \mathrm{ml}$ of urine or $\mathrm{ng} / \mathrm{mg}$ of creatinine are expressed as percent of values obtained with control, non-salttreated animals. Values shown are the averages \pm SE calculated from four different experiments.

\section{Discussion}

The initial reports of oxidative metabolism of arachidonic acid by microsomal cytochrome P450 suggested a role for the enzyme system in the bioactivation of this important fatty acid (24-26). The significance of these reactions was subsequently highlighted by the demonstration of stereospecific, in vivo, EET formation by various rat, rabbit, and human organs, and by the demonstration of microsomal cytochrome P450 as a member of the arachidonate metabolic cascade (5). Work from several laboratories, including ours, has documented the potent effects of several of the cytochrome $\mathrm{P} 450$ arachidonate metabolites in renal $\mathrm{Na}^{+}, \mathrm{K}^{+}$and $\mathrm{H}_{2} \mathrm{O}$ fluxes $(4,5)$. Additionally, a prohypertensive role for products of the cytochrome $\mathrm{P} 450$ arachidonic acid $\omega / \omega-1$ oxygenase was proposed, early on, by McGiff and collaborators (4).

In rats, excess dietary salt results in marked and selective increases in the activity of the renal cytochrome P450 epoxygenase (13). This metabolic response, as well as the documented effects of epoxygenase products on renal $\mathrm{H}_{2} \mathrm{O}$ and $\mathrm{Na}^{+}$transport and, in particular, the early demonstration of an inhibition of $\mathrm{Na}^{+}$reabsorption in the isolated cortical collecting tubule by EETs $(4,7)$ lead us to propose that the epoxygenase may be part of the adaptive response of the kidney to excess $\mathrm{Na}^{+}$intake. Clotrimazole and ketoconazole, powerful inhibitors of microsomal cytochrome P450 (18), have been used in the past to characterize the functional significance of the hemoprotein in several isolated cell or whole organ preparations $(4,27,28)$. We have shown, using rat liver microsomal fractions, that clotrimazole selectively inhibits the arachidonic acid epoxygenase and that it is a substantially less effective inhibitor of the arachidonic acid $\omega / \omega-1$ oxygenase reaction (18). The demonstration that Sprague-Dawley rats can be made hypertensive by the combined effects of salt loading and inhibition of their renal epoxygenase indicated a role for this enzyme system in renal
Table II. Isomeric Composition of the Epoxygenase Metabolites Present in the Urine of Salt-sensitive (DS) and Salt-resistant (DR) Rats

\begin{tabular}{ccc}
\hline Epoxygenase product & DR genotype & DS genotype \\
\hline & \% of total epoxygenase products \\
$5,6-$ & 23 & 22 \\
$8,9-$ & 15 & 17 \\
$11,12-$ & 17 & 22 \\
$14,15-$ & 44 & 39 \\
\hline
\end{tabular}

Urine samples were collected from male DS and DR animals exactly as in Fig. 5. After extraction and purification the concentration of each individual EET and DHET was determined by gas chromatography/ mass spectral technique as described (17). To calculate the percent distribution of the different epoxygenase products, the concentration of the each individual EET was added to that of the corresponding DHET positional isomer. Values shown are averages calculated from four different experiments with SE of the mean $\leq 15 \%$ of the averages.

$\mathrm{Na}^{+}$metabolism. However, as with most inhibitor studies, this interpretation must take into consideration other possible actions of clotrimazole, not directly associated with inhibition of the renal epoxygenase. Thus, for example, clotrimazole has been shown to inhibit steroid hydroxylases (29). Nevertheless, two well-known factors make it unlikely that the functional effects described are caused by inhibitor-dependent modifications in steroid production $(a)$ under conditions of excess dietary salt, as those used here, mineralocorticoid biosynthesis is suppressed, and $(b)$ adrenal steroid effects on blood pressure usually develop weeks after experimental manipulation of mineralocorticoid levels. Additionally, the demonstration that indomethacin, a potent inhibitor of prostanoid biosynthesis, had no significant effects on the MABP of salt-loaded animals provided strong evidence that, under our experimental conditions, the effects of clotrimazole were cyclooxygenase independent. Finally, initial spectral studies suggested that nitric oxide (NO) synthase may be a cytochrome P450 type protein $(30,31)$. However, subsequent amino acid sequence comparisons showed the lack of significant structural homologies between these proteins, including the absence in the NO synthase proteins of conserved cytochrome P450 domains essential for catalytic activity (32). More recently, it has been shown that clotrimazole does not inhibit the metabolism of L-arginine to L-citrulline by the NO synthase of endothelial cells (33) and that, in the isolated perfused rat kidney, the NO-dependent component of the vasodilator effect of bradykinin is not affected by clotrimazole (34).

Previous studies indicated that $(a)$ the renal epoxygenase ( $\mathrm{s}$ ) belong to the cytochrome P450 2C gene subfamily $(6,13,35$, 36 ), (b) cytochrome P450 2C23 is the predominant, constitutive, epoxygenase isoform in the rat kidney (35) and, (c) excess dietary salt induces a renal cytochrome P450 2C isoform that appears to be different than cytochrome P450 2C23 $(13,35)$. We have previously demonstrated by nucleic acid hybridization that neither the $4 \mathrm{~A} 1,4 \mathrm{~A} 2,2 \mathrm{C} 11$, or $2 \mathrm{C} 23$ genes are upregulated by excess dietary salt (35). More recently we expanded these studies using sequence specific synthetic oligonucleotides for cytochromes P450 4A1, 4A2, 2C6, 2C7, 2C11, 2C23, and 2C24 (30-40-mer each, containing published sequences from the untranslated 3 '-end of the corresponding cDNAs) and 
poly $(\mathrm{A})^{+}$RNAs isolated from the kidneys of control and salttreated rats. So far we have been unable to demonstrate a timedependent upregulation in the kidney levels of the corresponding mRNAs after salt loading. These results indicate that either the salt-inducible protein is a novel cytochrome $\mathrm{P} 450$ isoform or that, alternatively, protein induction occurs in the absence of demonstrable transcriptional regulation. Transcription-independent upregulation of microsomal cytochrome P450s has been demonstrated (37).

As an alternate approach to the inhibitor studies described above, we characterized the biochemical properties of the renal epoxygenase activity in control and salt-treated Dahl rats. The Dahl salt-sensitive (DS) and Dahl salt-resistant (DR) rat model of hypertension was developed in the early 1960's from Sprague-Dawley stocks by selective breeding techniques (21). In this rat model of salt-sensitive hypertension, genomic differences are responsible for functional differences in renal salt handling and the development of systemic hypertension in the DS genotype $(21,22)$. While in these animals, as in humans, hypertension is most likely a polygenic trait, transplantation studies clearly demonstrated that the kidney genotype played a pivotal role in the blood pressure response to increased dietary salt (22). Although the molecular basis underlining the saltsensitive hypertension phenotype are yet to be fully understood, in the past, several biochemical alterations have been documented in the DS rat, including alterations in the regulation of renal $\mathrm{Na}^{+}, \mathrm{K}^{+}$ATPases by dopamine $(38,39)$, the biosynthesis of mineralocorticoids $(40,41)$ and, the structure of the genes coding for renin (42) and $\mathrm{Na}^{+}, \mathrm{K}^{+}$ATPases (43, 44). The results reported here demonstrate that, as with normal SpragueDawley animals (13), DR rats responded to an increase salt intake by inducing the activity of their kidney cytochrome P450 AA epoxygenase and thus, upregulating the concentration of epoxygenase metabolites in the urine. On the other hand, saltloaded DS rats appeared unable to induce the activities of the renal epoxygenase and, as a consequence, the urinary concentration of their epoxygenase metabolites decreased. Hence, concomitant with increases in dietary salt intake, only the DR animals appeared capable of upregulating their epoxygenase activity and thus, prevent a fall in the urinary concentration of epoxygenase metabolites.

Our studies in two strains of rats, one in which hypertension can be induced during salt loading by inhibiting the arachidonate epoxygenase and, the other, the Dahl salt-sensitive model, in which a genetic predisposition to salt-dependent hypertension has been associated with a functional defect in the response of the renal arachidonate epoxygenase to excess dietary salt (13, 35 ), indicate an antihypertensive role for this enzyme system. Hence, it appears that an increased epoxygenase activity may prevent systemic hypertension in response to excess dietary salt. Indeed, one of the epoxygenase metabolites, 5,6-EET, is a powerful inhibitor of $\mathrm{Na}^{+}$absorption in the distal nephron (7). However, the details of the mechanisms by which the epoxygenase metabolites may regulate renal $\mathrm{Na}^{+}$and/or $\mathrm{H}_{2} \mathrm{O}$ homeostasis, as well as the molecular mechanism by which dietary salt regulates the biosynthesis of the arachidonate epoxygenase (s), remain to be established.

The importance of microsomal cytochrome P450 as the catalyst for the oxidative transformation of several xenobiotics is well established ( 45 and references therein). In addition to its recognized toxicological and pharmacological relevance, the last few years have witnessed a renewed interest in exploring and defining additional functional roles for this enzyme system (3-5, 46 and references therein). The present experimental evidence, as well as that of others (3-5), indicates that this enzyme system may be a significant component of the physiological mechanisms that control body fluid volume and composition. Indeed, it appears that either pharmacological inhibition or an inherited disorder of renal epoxygenase activity (or its regulation ) predisposes to salt-sensitive hypertension in rodents. These results, in conjunction with previous studies from several laboratories (3-5) provide decisive evidence that microsomal cytochrome $\mathrm{P} 450$, as a participant of the arachidonic acid metabolic cascade, plays significant functional roles in controlling tissue and body homeostasis. Current efforts are directed towards the identification of the kidney epoxygenase isoform( $\mathrm{s}$ ) responsive to excess dietary salt, as well as the molecular characterization of phenotypic differences in salt-dependent epoxygenase activities and/or regulation between the Dahl DR and DS genotypes. Since salt sensitivity is an important feature of hypertension in a large subpopulation of humans with essential hypertension, there are important clinical reasons for studying the molecular, catalytic, and genetic properties of the corresponding human homologues of the rat cytochrome P450 2C subfamily isoforms. We are confident that the results reported here will serve to further stimulate research in this clinically important area.

\section{Acknowledgments}

This work was supported by United States Public Health Services grant NIHDK 38226.

\section{References}

1. Needleman, P., J. Turk, B. A. Jakschik. A. R. Morrison, and J. B. Lef kowith. 1986. Arachidonic acid metabolism. Annu. Rev. Biochem. 55:69-102.

2. Smith, W. L. 1992. Prostanoid biosynthesis and mechanism of action. Am. J. Physiol. 263:F181-F191.

3. Fitzpatrick, F. A., and R. C. Murphy. 1989. Cytochrome P-450 metabolism of arachidonic acid: Formation and biological actions of "epoxygenase"-derived eicosanoids. Pharmacol. Rev. 40:229-241.

4. McGiff, J. C. 1991. Cytochrome P-450 metabolism of arachidonic acid. Annu. Rev. Pharmacol. Toxicol. 31:339-369.

5. Capdevila, J. H., J. R. Falck, and R. W. Estabrook. 1992. Cytochrome P450 and the arachidonate cascade. FASEB (Fed. Am. Soc. Exp. Biol.) J. 6:731-736.

6. Capdevila, J. H., A. Karara, D. J. Waxman, M. V. Martin, J. R. Falck, and F. P. Guengerich. 1990. Cytochrome P-450 enzyme-specific control of the regioand enantiofacial selectivity of the microsomal arachidonic acid epoxygenase. $J$. Biol. Chem. 265:10865-10871.

7. Jacobson. H. R.. S. Corona. J. H. Capdevila. N. Chacos, S. Manna, A. Womack, and J. R. Falck. 1984. Effects of epoxyeicosatrienoic acids on ion transport in the rabbit cortical collecting tubule. In Prostaglandins and Membrane Ion Transport. P. Braquet, R. P. Garay, J. C. Frolich, and S. Nicosia, editors. Raven Press, New York. 311-318.

8. Romero, M. F., Z. Madhun. U. Hopfer, and J. G. Douglas. 1990. An epoxygenase metabolite of arachidonic acid 5,6 epoxy-eicosatrienoic acid mediates angiotensin-induced natriuresis in proximal tubule epithelium. In Advances in Prostaglandin. Thromboxane and Leukotriene Research. B. Sammuelson, P. Ramwell, R. Paoletti, and G. Folco, editors. Raven Press, New York. 21:205208.

9. Schlondorff, D.. E. Petty, J. A. Oates, and S. D. Levine. 1987. Epoxygenase metabolites of arachidonic acid inhibit vasopressin response in toad bladder. Am. J. Physiol. 253:F464-F470.

10. Hirt, D. L., J. H. Capdevila, J. R. Falck. M. Breyer, and H. R. Jacobson. 1989. Cytochrome P450 metabolites of arachidonic acid are potent inhibitors of vasopressin action on rabbit cortical collecting duct. J. Clin. Invest. 84:18051812

11. Katoh, T., K. Takahashi, J. H. Capdevila, A. Karara, J. R. Falck, H. R. Jacobson, and K. F. Badr. 1991. Glomerular stereospecific synthesis and hemodynamic actions of 8,9-epoxyeicosatrienoic acid in rat kidney. Am. J. Physiol. 261:F578-F586. 
12. Satoh, T., H. T. Cohen, A. I. Katz. 1993. Intracellular signaling in the regulation of renal Na-K-ATPase. II. Role of eicosanoids. J. Clin. Invest. 91:409 415.

13. Capdevila, J. H., S. Wei, J. Yang, A. Karara, H. R. Jacobson, J. R. Falck F. P. Guengerich, and R. N. Dubois. 1992. Cytochrome P450 arachidonic acid epoxygenase: Regulatory control of the renal epoxygenase by dietary salt loading. J. Biol. Chem. 267:21720-21726.

14. Iwai, N., and T. Inagami. 1990. Isolation of preferentially expressed genes in the kidneys of hypertensive rats. Hypertension. 17:161-169.

15. Catella, F., J. A. Lawson, D. J. Fitzgerald, and G. A. Fitzgerald. 1990. Endogenous biosynthesis of arachidonic acid epoxides in humans: Increased formation in pregnancy-induced hypertension. Proc. Natl. Acad. Sci. USA. 87:58935897.

16. Capdevila, J. H., J. R. Falck, E. Dishman, and A. Karara. 1990. Cytochrome P-450 arachidonate oxygenase Methods Enzymol. 187:394-394.

17. Capdevila, J. H., E. Dishman, A. Karara, and J. R. Falck. 1991. Cytochrome P450 arachidonic acid epoxygenase: Stereochemical characterization of epoxyeicosatrienoic acids. Methods Enzymol. 206:441-453.

18. Capdevila, J. H., L. Gil, M. Orellana, L. J. Marnett, I. J. Mason, P. Yadagiri, and J. R. Falck. 1988. Inhibitors of cytochrome P-450-dependent arachidonic acid metabolism. Arch. Biochem. Biophys. 261:257-263.

19. Gardiner, S. M., P. A. Kemp, R. M. Palmer, and S. Moncada. 1992. Nitric oxide synthase inhibitors cause sustained, but reversible, hypertension and hindquarters vasoconstriction in Brattleboro rats. Eur. J. Pharmacol. 213:449451 .

20. Rees, D. D., R. M. Palmer, R. Schulz, H. Hodson, and S. Moncada. 1990. Characterization of three inhibitors of endothelial nitric oxide synthase in vitro and in vivo. Br. J. Pharmacol. 101:746-752.

21. Rapp, J. P. 1982. Dahl salt-susceptible and Dahl salt-resistant rats. A review. Hypertension. 4:753-763.

22. Dahl, L. K., M. Heine, and K. Thompson. 1974. Genetic influence of the kidneys on blood pressure. Evidence from chronic renal homografts in rats with opposite predisposition to hypertension. Circulation Res. 34:94-101.

23. Roman, R. J., Y. H. Ma, B. Frolich, and B. Markham. 1993. Clofibrate prevents the development of hypertension in Dahl salt-sensitive rats. Hyperten sion. 21:985-988.

24. Capdevila, J., N. Chacos, J. Werringloer, R. A. Prough, and R. W. Estabrook. 1981. Liver microsomal cytochrome P-450 and the oxidative metabolism of arachidonic acid. Proc. Natl. Acad. Sci. USA. 78:5362-5366.

25. Oliw, E. H., and J. A. Oates. 1981. Oxygenation of arachidonic acid by hepatic microsomes of the rabbit. Mechanism of biosynthesis of two vicinal dihydroxyeicosatrienoic acids. Biochim. Biophys. Acta. 666:327-340.

26. Morrison, A. R., and N. Pascoe. 1981. Metabolism of arachidonic acid through NADPH-dependent oxygenase of renal cortex. Proc. Natl. Acad. Sci. USA. 78:7375-7378.

27. Junier, M. P., F. Dray, I. Blair, J. H. Capdevila, E. Dishman, J. R. Falck, and S. R. Ojeda. 1989. Epoxygenase products of arachidonic acid are endogenous constituents of the hypothalamus involved in $\mathrm{D}_{2}$ receptor-mediated, dopamineinduced release of somatostatin. Endocrinology. 126:1534-1540.

28. Alvarez, J., M. Montero, and J. Garcia-Sancho. 1992. Cytochrome may regulate plasma membrane $\mathrm{Ca}^{2+}$ permeability according to the filling state of the intracellular $\mathrm{Ca}^{2+}$ stores. FASEB (Fed. Am. Soc. Exp. Biol.) J. 6:786-792.

29. Wada, A., T. Ohnishi, Y. Nonaka, and M. Okamoto. 1988. Inhibition of bovine adrenocortical mitochondrial cytochrome $\mathrm{P}-450_{11 \beta}$-mediated reactions by imidazole derivatives and mineralocorticoid analogs. J. Steroid Biochem. 31:803808.

30. White, K. A., and M. A. Marletta. 1992. Nitric oxide synthase is a cytochrome P-450 type hemoprotein. Biochemistry. 31:6627-6631.

31. McMillan, K., D. S. Bredt, D. J. Hirsch, S. H. Snyder, J. E. Clark, and B. S. S. Masters. 1992. Cloned, expressed rat cerebellar nitric oxide synthase contains stoichiometric amounts of heme, which bind carbon monoxide. Proc. Natl. Acad. Sci. USA. 89:11141-11145.

32. Nelson, D. R., T. Kamataki, D. J. Waxman, F. P. Guengerich, R. W. Estabrook, R. Feyereisen, F. J. Gonzalez, M. J. Coon, I. C. Gunsalus, O. Gotoh, K. Okuda, and D. W. Nebert. 1993. The P450 superfamily: Update on new sequences, gene mapping, accession numbers, early trivial names of enzymes and nomenclature. DNA Cell Biol. 12:1-51.

33. Oyekan, A. O., J. C. McGiff, W. P. Rosencrantz, and J. Quilley. 1994. Relaxant responses of rabbit aorta: Influence of cytochrome P450 inhibitors. $J$. Pharmacol. Exp. Ther. 268:262-269.

34. Fulton, D., J. C. McGiff, and J. Quilley. 1992. Contribution of NO and cytochrome $\mathrm{P} 450$ to the vasodilator effect of bradykinin in the rat kidney. $\mathrm{Br}$. J. Pharmacol. 107:722-725.

35. Karara, A., K. Makita, H. R. Jacobsson, J. R. Falck, F. P. Guengerich, R. N DuBois, and J. H. Capdevila. 1993. Molecular cloning, expression, and enzymatic characterization of the rat kidney cytochrome P-450 arachidonic acid epoxygenase. J. Biol. Chem. 268:13565-13570.

36. Laethem, R. M., C. L. Laethem, and D. R. Koop. 1992. Purification and properties of a cytochrome $\mathrm{P} 450$ arachidonic acid epoxygenase from rabbit renal cortex. J. Biol. Chem. 267:5552-5559.

37. Gonzalez, F. J. 1989. The molecular biology of cytochrome P450s. Pharmacol. Rev. 40:243-286.

38. Nishi, A., A. M. Bertorello, and A. Aperia. 1992. High salt diet downregulates proximal tubule $\mathrm{Na}^{+}, \mathrm{K}^{+}$-ATPase activity in Dahl salt-resistant but not in Dahl salt-sensitive rats: Evidence of defective dopamine regulation. Acta Physiol. Scand. 144:236-267.

39. Satoh, T., H. T. Cohen, and A. I. Katz. 1993. Different mechanisms of renal Na-K-ATPase regulation by protein kinases in proximal and distal nephron Am. J. Physiol. 265:F399-F405.

40. Rapp, J. P., and L. K. Dahl. 1976. Mutant forms of cytochrome P-450 controlling both 18 -and 11 beta steroid hydroxylation in the rat. Biochemistry. 15:1235-1242.

41. Matsukawa, N., Y. Nonaka, J. Higaki, M. Nagano, H. Mikami, T. Ogihara, and M. Okamoto. 1993. Dahl's salt-resistant normotensive rat has mutation in cytochrome $\mathrm{P}^{4} 50_{11 \beta}$, but the salt-sensitive hypertensive rat does not. J. Biol. Chem. 268:9117-9121.

42. Rapp, J. P., S. M. Wang, and H. Dene. 1989. A genetic polymorphism in the renin gene of the Dahl rats cosegregates with blood pressure. Science (Wash. DC). 243:542-544.

43. Herrera, V. L. M., and N. Ruiz-Opazo. 1990. Alteration of $\alpha 1 \mathrm{Na}^{+}, \mathrm{K}^{+}-$ ATPase ${ }^{86} \mathrm{Rb}$ influx by a single amoino acid substitution. Science (Wash. DC). 249:1023-1026.

44. Simonet, L., E. Lezin, and T. W. Kurtz. 1991. Sequence analysis of the $\alpha 1 \mathrm{Na}^{+}, \mathrm{K}^{+}$-ATPase gene in the Dahl salt-sensitive rat. Hypertension. 18:689693.

45. Guengerich, F. P. 1991. Reactions and significance of cytochrome P-450 enzymes. J. Biol. Chem. 266:10019-10022.

46. Schenkman, J. B., K. E. Thummel, and L. V. Favreau. 1989. Physiological and pathophysiological alterations in rat hepatic cytochrome P-450. Drug Metab. Rev. 20:557-584. 